\section{Carolina de Weerth Jan K. Buitelaar}

\title{
Childbirth complications affect young infants' behavior
}

Abstract Background The process of childbirth and its complications have been related to the newborn's condition and to development at later ages. In this study, we examine how mode of delivery and delivery complications are related to the behavior and cortisol reactivity of infants during the first 2 months. Methods Delivery factors (i.e. mode, duration, fetal heart function, and 5-min Apgar score) were determined in 116 healthy, term, firstborn infants. The infants' behavioral and cortisol reactivity to stressors were assessed during a physical examination at 11 days and an inoculation at 2 months. Daily behavior at 6 weeks was followed by means of a four-day parental diary. Results Indicators of a more stressful delivery were linked to more crying/fussing in the infant and to more difficulties in regulating the infants' behavior (i.e., more unsoothability and longer crying bouts). Specific delivery complications rather than mode of delivery were linked to infants' behavior. No significant associations however, were found between childbirth and the infants' cortisol reactivity to a physical examination and an inoculation. Conclusions Childbirth complications, even when mild, can apparently affect early infant behavior. These effects extend over different types of situations, and last at least until the age of 2 months, and should be taken into account in studies on infant behavior. Earlier findings of relations between childbirth complications and infant cortisol reactivity could not be replicated in this study, possibly due to the confounding effect of parity, and to the important intra-individual variability present in the infants' cortisol.

Key words infants childbirth - cortisol stress reactivity - behavior

\section{Introduction}

Gestation finalizes with delivery, a process that by itself can be an important stressor for the fetus [6]. Childbirth factors as mode of delivery, length of labor, and signs of fetal stress have been found to influence the newborn's condition after birth. For example, neonates born by elective caesarean section have higher rates of respiratory morbidity, possibly because labor and delivery produce a surge of catecholamines in the fetus, which is in turn important for postnatal lung adaptation [26]. Also, meconium staining of the amniotic fluid is significantly associated with generally poorer neonatal outcome [27]. Moreover, childbirth factors have been related to neonatal development as well as development far beyond the neonatal period $[10,21]$. 
Childbirth as a stressful process for the fetus may also influence the endocrinological stress status of newborns. Stress hormones measured immediately after birth have shown assisted vaginal deliveries to be more stressful [6] or equally stressful [16] than normal vaginal deliveries. In turn, both are more stressful than elective caesarean sections $[6,16]$. After a few hours, however, the cortisol levels of elective caesarean-section newborns reach the levels of newborns delivered after spontaneous labor [17].

The delivery mode and the neonate's condition after birth have also been related to the infant's subsequent behavior and stress reactivity. Eight-week-old infants delivered by elective caesarean section showed significantly smaller cortisol and crying responses to an inoculation than infants delivered by normal and assisted vaginal deliveries [24]. Further, infants delivered by assisted deliveries showed significantly larger cortisol reactions than those delivered by normal vaginal deliveries. However, the same research group could not replicate these findings of relations between delivery mode and cortisol reactions to inoculations in a later, larger study [16]. Also, Keenan et al. [11] were unable to document any association between delivery complications and cortisol reactivity to stressors during the first $48 \mathrm{~h}$ of life, although behavioral reactivity was related to delivery mode in this study. In line with these findings, infants from assisted deliveries showed the longest durations of daily crying at 3 months of age, and those from elective caesareans the shortest [12].

Although delivery mode is often the result of emerging intrapartal complications, it constitutes a rather global characterization of the partus that may be less closely associated with the neonate's condition than specific delivery complications such as long duration of labor or deteriorated fetal heart function are. This is in line with Miller et al.'s study [16] in which a positive correlation was found between cord arterial cortisol at birth and later cortisol reactivity to inoculation, but not between mode of delivery and cortisol reactivity. The authors conclude that it is probably the degree of physical stress experienced at birth, rather than the mode of delivery per se, that is important. Therefore, more direct indicators of the neonate's condition and stress status at birth, as for example cortisol levels or Apgar scores, may be more related to subsequent cortisol reactivity than childbirth complications. Indeed, Ramsay and Lewis [19] found that an optimal birth condition (based on head circumference and Apgar score) was related to a high cortisol response to inoculation at 2 months and a low response at 6 months.

Extending the findings that pain and stress during delivery can have long-lasting consequences on infant behavior and physiology, Taddio and colleagues [22,
23] showed that experiencing pain during the first hours or days following the delivery can also affect infants' reactions to future painful stimuli. Newborns that had been circumcised or had been exposed to repeated heel lances reacted with more negative and stronger behavioral responses to subsequent stressors than infants that had not undergone the procedures. According to these authors, early experiences of pain such as of a circumcision may induce long-lasting changes in pain behavior because of alterations in the infant's central neural processing of painful stimuli. The pain would induce a sustained state of central neural sensitization or hyperexcitability that amplifies subsequent noxious neural input. These effects could be long lasting, as shown by the infants' behavioral reactions several months after the stressors. A comparable mechanism could be hypothesized to underlie the link between childbirth complications, as markers of a serious early life stressor, and the offspring's later behavioral reactions to stressors. Similarly to its effects on behavior, as in animal models, there are indications that an early stressful experience can also program the HPA axis in humans [16]. The central processing of stressful stimuli mostly produces effects both on a physiological and behavioral/emotional level. However, these two types of reactions to stress are not always correlated in adults [20]; in other words, researchers measuring both reactions to stressors, do not always find straightforward relations between both. In infants, behavior and cortisol reactions are often modestly correlated, but not always, and not always in the same direction $[9,11,13,14]$. Because both types of reactions constitute different aspects of the stress reaction, and because both are related to an individual's health and adaptation, it is important to study the effects of stressors on both types of reactions.

In summary, the literature suggests that childbirth complications can influence the infant's behavior and cortisol reactivity during the first months after birth. However, the direction of the association between childbirth complications and neonatal condition, and early cortisol reactivity remains unclear: from greater to smaller reactivity, or no association at all. These differences are most likely at least partly due to diversity of study designs.

The purpose of the present study was therefore to further investigate these issues by following a population of healthy, term infants, born after relatively uncomplicated deliveries. By looking at both delivery mode and specific mild complications (i.e., normal variation within delivery factors of uncomplicated births), and by assessing the infants physiologically and behaviorally, and at three different ages, we aimed at obtaining a more complete picture of the relations between childbirth complications and infant development than that of earlier studies. We 
hypothesized that in a normal population, infants born with assisted and more complicated deliveries, and in less optimal condition would display greater irritability (i.e., crying/fussing) with stressors and in daily life. Because of the conflicting findings with respect to cortisol reactivity, we chose not to formulate specific hypotheses on this topic, but to attempt to clarify it further by linking childbirth complications to the infant's cortisol reactions to both a physical examination and an inoculation. We further expected the effects to be visible throughout the first 2 months (i.e., at the three different ages studied), and to find no or a modest relation between the cortisol and behavioral reactions to stressors.

\section{Method}

\section{Participants}

The participants were 116 Caucasian women and their healthy firstborn infants. The women were recruited early in pregnancy, through folders distributed by their midwives. Of the 146 women that began the study, 24 were excluded because of health problems, medication use, severe pregnancy and/or childbirth complications, twinning, and prematurity. A further six discontinued the study during the pregnancy due to lack of time or interest. These women did not differ from the remaining group in their demographic data.

The remaining 116 participants were healthy, and had normal, uncomplicated singleton pregnancies with term deliveries ( $>37$ weeks). Their mean age at inclusion was 31.2 years $(S D=3.7)$, and their marital status: $93.1 \%$ married/cohabiting, $2.6 \%$ single, and $4.3 \%$ got married or started cohabiting during the research period. The participants' educational levels were: $0.9 \%$ primary (elementary), $21.8 \%$ secondary, $43.5 \%$ tertiary (post-secondary or higher education), and $33.9 \%$ university; $92.2 \%$ of the women had a paid job during the pregnancy. The infants (50\% girls) were delivered after a mean of 40.2 weeks $(S D=1.3)$, had a mean birth weight of $3,493.1 \mathrm{~g}(\mathrm{SD}=499.3$, minimum $=2,470 \mathrm{~g}$, maximum $=4,800 \mathrm{~g}$ ), a mean 1 min Apgar of $8.7(S D=1.0, \min =5, \max =10)$ and a mean 5-min Apgar of $9.7(\mathrm{SD}=0.6, \quad \min =7$, $\max =10$ ). Male infants are not routinely circumcised in the Netherlands, except for religious reasons. The families participating in this study did not belong to religions in which male infants are circumcised.

\section{Procedure}

Delivery mode and childbirth complications were studied in relation to the behavioral and cortisol reactivity to a physical examination at 11 days and an inoculation at 2 months, and to 6-week behavioral diary recordings.

\section{Measures: independent variables}

Delivery mode. Of the 116 infants, 68 were born by normal vaginal delivery, 34 had an assisted delivery (forceps or ventouse), 12 were born by emergency caesarean, and two by elective caesarean. This last group of infants was so small that it was excluded from the analyses. Although the dependent variables had some missing data, there was no selective attrition for mode of delivery (the minimum representation for the emergency caesarean group was for the inoculation: eight infants for the cortisol, nine for the behavior).

Specific delivery complications. Four variables were included: duration of first stage, duration of second stage, fetal heart function, and 5-min Apgar score (the Apgar score is used to evaluate the condition of a neonate; ratings of 0,1 , or 2 are given for color, heart rate, response to stimulation of the sole of the foot, muscle tone, and respiration, with 10 being a perfect score). A score of 0 was assigned when the infant scored optimally on the variable, and a 1 when the score was less optimal. The infants' scores on these four variables were uncorrelated. The optimal and less optimal categories for stage durations and Apgar score were created solely for research purposes so that in this clinically healthy population around 25\% would fall in the less optimal category (see Table 1). Thus, the categorization of "less optimal" has no clinical value whatsoever for these variables. Table 2 presents the distribution and mean values of the study population, according to delivery mode and specific complications.

\section{Measures: dependent variables}

Physical examination. The infants' behavioral and cortisol reactivity to a neurological examination [18] were assessed at home at 11.5 days of age (Standard Deviation SD: 2.3). The examinations took place either in the morning or afternoon. This did not affect cortisol levels, as at this age the circadian rhythm is not present [5]. The first author performed the examination, which had a mean duration of $19.9 \mathrm{~min}$ (SD: 3.5). The examination, which took place on a changing mat, commenced when the infant was nondistressed or sleeping, and slowly increased in intensity of stimulation. If the infant fussed/cried during the examination, the experimenter soothed him/her in a routine manner: first by stroking/talking, and then by picking up and rocking gently. As soon as the infant calmed, the examination was continued. 
Table 1 Variables used to determine optimal and less optimal delivery conditions

\begin{tabular}{lll}
\hline Variable & Optimal $(=0)$ & Less optimal $(=1)$ \\
\hline Duration of first stage (dilatation) & $<=11.75 \mathrm{~h}(75 \%)$ & $>11.75 \mathrm{~h}$ \\
Duration of second stage (pushing) & $<=1.5 \mathrm{~h}(78 \%)$ & $>1.5 \mathrm{~h}$ \\
Fetal heart function & Normal pattern (82\%) & Non-reassuring pattern \\
Apgar score, $5 \mathrm{~min}$ & $10(76 \%)$ & $<10$ \\
\hline
\end{tabular}

Notes: the percentage of the infants that scored optimally is presented between brackets. The fetal heart function was scored as: normal (FHR normal during the whole delivery or showing a slight tachy- or bradycardia, and only for a short period of time) or non-reassuring pattern (FHR showing recurrent and/or longlasting tachy- or bradycardia, often unresponsive to medical management)

Table 2 Descriptive values and distribution of the study population according to delivery mode and specific delivery complications

\begin{tabular}{|c|c|c|c|c|c|c|c|c|c|}
\hline \multirow{3}{*}{\multicolumn{2}{|c|}{ Specific delivery variables }} & \multicolumn{8}{|c|}{ Delivery mode } \\
\hline & & \multicolumn{2}{|c|}{$\begin{array}{l}\text { Vaginal } \\
(n=68)\end{array}$} & \multicolumn{2}{|c|}{$\begin{array}{l}\text { Assisted } \\
(n=34)\end{array}$} & \multicolumn{2}{|c|}{$\begin{array}{l}\text { Emergency } \\
\text { caesarean }(n=12)\end{array}$} & \multicolumn{2}{|c|}{$\begin{array}{l}\text { All deliveries } \\
(n=114)\end{array}$} \\
\hline & & Mean & SD & Mean & SD & Mean & SD & Mean & SD \\
\hline \multirow{4}{*}{\multicolumn{2}{|c|}{$\begin{array}{l}\text { Duration of first stage (dilatation) }{ }^{\text {a }} \\
\text { Duration of second stage (pushing) } \\
\text { Apgar score, } 5 \text { minutes }\end{array}$}} & 8.06 & 4.94 & 10.45 & 4.65 & 12.04 & 6.70 & 9.18 & 5.23 \\
\hline & & 0.94 & 0.55 & 1.66 & 0.77 & 1.42 & 1.01 & 1.19 & 0.72 \\
\hline & & 9.78 & 0.57 & 9.53 & 0.66 & 9.75 & 0.45 & 9.70 & 0.59 \\
\hline & & Count & Row \% & Count & Row \% & Count & Row \% & Count & Group \% \\
\hline \multirow[t]{2}{*}{ Fetal heart function } & Normal & 63 & $67.0 \%$ & 24 & $25.5 \%$ & 7 & $7.4 \%$ & 94 & $82.5 \%$ \\
\hline & Non-reassuring & 5 & $25.0 \%$ & 10 & $50.0 \%$ & 5 & $25.0 \%$ & 20 & $17.5 \%$ \\
\hline
\end{tabular}

${ }^{a}$ Means and standard deviations in hours

The infant's cortisol reaction was assessed by sampling saliva upon arrival (sample 1), and $40 \mathrm{~min}$ and $60 \mathrm{~min}$ after the beginning of the examination (samples 2 and 3, respectively). These times were chosen because it takes around 25 min for the effects of a stressor to be noticeable in saliva cortisol [8]. Thus, sample 1 constituted the basal cortisol, sample 2 the infant's reaction to the stressor (at around 15 min into the examination), and sample 3 the poststressor situation (at around $15 \mathrm{~min}$ of being with the caregiver again). Complete cortisol series were obtained in 108 infants. The following cortisol reactivity dependent variables were computed: stress (delta: sample 2 minus sample 1), and recovery (sample 3 minus sample 1). The choice of these change scores as measures for stress and recovery is based on commonly used methodology for cortisol reactivity and recovery analyses (see [15], for a discussion on advantages and disadvantages).

The infant's behavioral reactions were observed from videotapes taken during the examination. The videotapes of 107 infants were adequate for scoring. The following behaviors were scored: fussing (discrete squeaks, fretting, whining, whimpering), crying (weeping; higher arousal level than when fussing, more muscle tension, often accompanied by intense facial coloring), soothing (examiner attempts to calm infant: talks, touches, pats, rocks softly in arms), and pacifier or finger (of the examiner-as part of the testing-or of the infant in the infant's mouth). The pacifier was seldom used, and then as a means of calming an infant that was otherwise unsoothable.

All behaviors were scored continuously, except for fussing that was scored with interval scoring (5-s intervals). A research assistant scored the videotapes, after reaching an adequate inter-observer reliability with the first author (mean percentage agreement over 10 infants was $76.6 \%$ ). The following behavioral reactivity dependent variables were computed: crying ( $\%$ of examination time), latency to cry (in seconds), fussing (\% of 5-s observation periods), soothing (\% of examination time), and pacifier (\% of examination time).

Daily behavior. When the infants were 6.1 weeks old (SD: 0.4), their mothers kept the 24-h Baby Day Diary [1] for four consecutive days. This lengthy period was chosen in order to avoid otherwise unreliable data due to intra-individual variability in crying/fussing [2]. A total of 102 mothers filled in usable diaries (also included are five that had adequate data on three out of the 4 days). The following behavioral dependent variables were computed: crying and fussing, separately (mean daily duration, daily frequency, and bout duration), and unsoothable crying-defined as crying that the caregivers were not able to stop through soothing efforts-(due to its very low frequency, only mean daily duration was used).

Inoculation. The infants received their first two inoculations (Diptheria-pertussis-tetanus-polio, and 
Haemophilus influenza type b) between $0840 \mathrm{~h}$ and $1645 \mathrm{~h}$, at an age of 65.6 days (SD: 12.5). It was not possible for researchers to be present during the inoculations, and therefore, after receiving clear written instructions the mothers themselves collected the inoculation materials. On a form they filled in the infant's feeding and sleeping times of the inoculation day, the inoculation time, and when the second saliva sample was due. The inoculations took place at a Well Baby Clinic, and the nurse/doctor performing the inoculation answered three questions on the infant's behavior. Once at home, the mothers sent all the inoculation materials by post to the lab, where the saliva samples were placed at $-18^{\circ} \mathrm{C}$ until analysis. Complete cortisol data were obtained from 81 infants and questionnaire data were obtained for 91 infants; there was no selective attrition for delivery mode and complications. The infant's cortisol reaction was assessed by sampling saliva upon arrival (sample 1, basal cortisol), and $25 \mathrm{~min}$ after receiving the inoculations (sample 2, stress cortisol). The cortisol reactivity dependent variable used was: stress (delta: sample 2 minus sample 1 ).

The infant's behavioral reactions to the inoculation were assessed through the following three questions: "How much did the infant cry?", "How loud did the infant cry?", and "How soothable was the infant?" (7point Likert scales, respectively: "not at all-very much," "softly-loudly," "easy to sooth-difficult to sooth").

\section{Potential confounders}

For both stressors, the following variables were scored as possible confounders: type of feeding (breast versus bottle), minutes since last feeding, minutes awake, duration of the last sleep, age in days, time of day (control for cortisol circadian rhythm), birth weight, length, head circumference, and duration of pregnancy. Also, the infant's state prior to the inoculation was categorized as asleep/non-distressed or fussing/crying. For the diary data, the following variables were scored: type of feeding, age in days, birth weight, length, head circumference, and duration of pregnancy.

\section{Saliva samples and cortisol determination}

The infant's saliva was collected by swabbing the child's mouth with a cotton dentist roll for a few minutes. The roll was then placed in a disposable $5 \mathrm{ml}$ syringe, closed with a small plastic cap. The samples were kept at $-18^{\circ} \mathrm{C}$ for periods of up to several weeks, until the moment of analysis. In order to avoid the loss of data due to insufficient saliva, the samples were analyzed in duplo using an extraction method specially designed for small quantities of saliva [4]. To control for interassay variation, the cortisol assays were performed with all samples of the same infant in the same batch. Cortisol was determined with an in house competitive RIA (modified for saliva from 25), which is insensitive to breast milk and formula contamination [4], and where the sensitivity of the direct assay was $0.5 \mathrm{nmol} / \mathrm{l}$; within-assay variability was $4 \%$ at $10 \mathrm{nmol} / \mathrm{l}$, and between-assay variation was $9 \%$ at $4 \mathrm{nmol} / \mathrm{l}$ and $5 \%$ at $10 \mathrm{nmol} / \mathrm{l}$.

\section{Statistical analyses}

ANOVA's were used to analyze the relations between the independent and dependent variables. First, correlations were calculated between potential confounders (sleep, feeding, time of day, etc.) and dependent variables. Only the confounders that correlated significantly $(P \leq 0.05)$ with a specific dependent variable were included in this variable's ANOVA's. In the results section, estimated marginal means and standard errors of the dependent variables are presented when confounders are involved, and means \pm SE when no confounders are involved. The delta cortisol for the physical examination was not normally distributed and was therefore normalized with square root prior to analysis. Non-parametric tests were used for analyses when variables could not be normalized, or when ANOVA's had non-normal residuals or non-homogenous variances. These tests were applied, when relevant, on the residuals obtained after linearly regressing the variable against the significant confounders. Corrections for multiple testing were not carried out because of the exploratory nature of the study.

\section{Results}

\section{Preliminary analyses}

\section{Effects of confounders}

Because not all confounders were normally distributed, their relations with the dependent variables were determined with non-parametric tests with two-tailed significances (Mann-Whitney $U$ tests and Spearman correlations). At the moment of the physical examination, $90 \%$ of the infants were breastfed and $10 \%$ were exclusively on formula; at the moment of the inoculation, these percentages were $68 \%$ and $32 \%$. The type of feeding the infants received had no influence on the dependent variables.

Physical examination. The cortisol variables were positively correlated to the "minutes since last feeding": stress (rho $=0.42, P<0.001$ ) and recovery (rho $=0.38, P<0.001)$. "Minutes awake" was nega- 

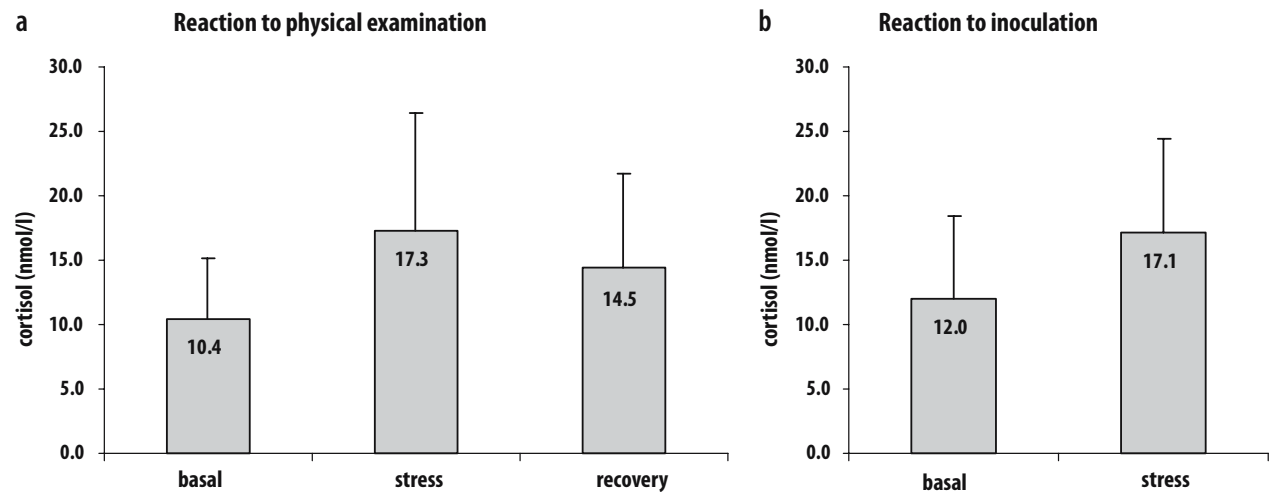

Fig. 1 Raw salivary cortisol levels (unadjusted for confounders) sampled during the physical examination at 11 days of age, $n=108$ (A) and the first inoculation at 2 months of age, $n=81$ (B). Error bars are standard deviations. All samples within each graph differ significantly from each other. Reaction to physical examination: sample 2 is taken 40 min after commencing the examination and therefore corresponds to around 15 min of ongoing examination; sample 3 is taken 60 min after commencing the examination and therefore corresponds to around $15 \mathrm{~min}$ after finalizing the examination. Reaction to inoculation: sample 2 is taken 25 min after the second vaccination is applied and therefore corresponds to the pain stimulus

tively correlated to recovery ( rho $=-0.22, P=0.02$ ), and "age in days" positively to both stress (rho $=0.21$, $P=0.03)$ and recovery $($ rho $=0.26, P=0.01)$. With respect to the behavioral reaction to the examination, "minutes since last feeding" was related to more use of the pacifier (rho $=0.24, P=0.01$ ), and "minutes awake" was related to more crying (rho $=0.21$, $P=0.03$ ), a shorter latency to cry (rho $=-0.36$, $P<0.001$ ), and more fussing (rho $=0.24, P=0.01$ ). "Duration of the last sleep" was negatively correlated to soothing ( $\mathrm{rho}=-0.20, P=0.05$ ), and finally, "duration of pregnancy" was negatively correlated to fussing (rho $=-0.19, P=0.05$ ).

Daily behavior. The results showed negative correlations between "age in days" and crying duration (rho $=-0.25, P=0.01$ ), crying frequency (rho $=$ $-0.22, P=0.02$ ), and unsoothable crying duration (rho $=-0.20, P=0.05$ ). The mean length of the crying bout was positively correlated to "birth weight" (rho $=0.23, P=0.02$ ), "birth length" (rho $=0.26$, $P=0.01)$ and "head circumference" (rho $=0.22$, $P=0.05)$.

Inoculation. The confounders were not correlated to the cortisol reactivity to the inoculation. The behavioral reactions to the inoculation showed "birth length" to be negatively correlated to crying amount (rho $=-0.24, P=0.03$ ). The infants' behavior prior to the inoculation (i.e., 69 infants were asleep/nondistressed and 20 infants were fussing/crying) was not related to their behavior after the inoculation.

\section{Main analyses}

The results will be presented as follows. First we describe the infants' behavioral and cortisol reactivity to the stressors. Next, we report on the associations between childbirth complications and infants' behavior, and finally on the associations between childbirth complications and infants' cortisol.

\section{Cortisol and behavioral reactivity to the stressors}

The infants' mean cortisol levels as a reaction to the stressors are shown in Fig. 1. The physiological reactions to the stressors were relatively large and significant. In the physical examination, all three samples differed significantly from each other (Friedman test, Chi-square $=42.13, P<0.001$; Wilcoxon Signed Ranks tests for each pair of samples, $z \leq-4.61, P<0.001$ ), and in the inoculation, both samples differed significantly from one another (Wilcoxon Signed Ranks test, $z=-4.69, P<0.001$ ). The stress and recovery cortisol variables of the physical examination were not correlated to the stress cortisol variable of the inoculation. The typical behavioral reactions to the stressors were an appearance and often increase of negative vocalizations during the course of the physical examination, and crying after the inoculation. The cortisol reactions to the stressors were not significantly correlated to the behavioral reactions to the stressors.

\section{Childbirth complications and behavior}

Delivery mode. Out of the 16 dependent variables of infant behavior, only pacifier use during the physical examination was related to mode of delivery $\left(F_{2,100}=5.98, P=0.001\right.$, effect of "mode of delivery": $P=0.01)$. Post hoc LSD tests showed that the percentage of examination time in which the infants had to be soothed with a pacifier/finger was higher in the infants born with assisted deliveries (estimated marginal mean \pm SE, $8.9 \pm 1.0$ ) than in those delivered with normal vaginal deliveries $(5.3 \pm 0.7 ; P=0.01)$ and with emergency caesarean sections $(5.0 \pm 1.7 ; P=0.03)$. 
Table 3 Estimated marginal means and standard errors of the behavioral reactions to the physical examination for the optimal and less optimal 5min Apgar groups

\begin{tabular}{|c|c|c|c|c|}
\hline \multirow[b]{2}{*}{ Behavior } & \multicolumn{2}{|l|}{ 5-min Apgar } & \multirow[b]{2}{*}{ Test statistic } & \multirow[b]{2}{*}{$P^{a}$} \\
\hline & $\begin{array}{l}<10 \\
(n=27) \\
\text { Mean } \pm \text { SD }\end{array}$ & $\begin{array}{l}10 \\
(n=89) \\
\text { Mean } \pm \text { SD }\end{array}$ & & \\
\hline Crying & $21.5 \% \pm 3.1$ & $15.8 \% \pm 1.8$ & $F_{1,103}=3.4, P<0.05$ & $<0.05$ \\
\hline Latency to cry & $327.9 s \pm 83.0$ & $617.0 s \pm 47.6$ & $F_{1}=7.8, P=0.001$ & $<0.01$ \\
\hline Fussing & $36.1 \% \pm 2.9$ & $28.0 \% \pm 1.7$ & $F_{1,102}=4.1, P<0.01$ & $<0.05$ \\
\hline Soothing & $15.9 \% \pm 1.8$ & $10.8 \% \pm 1.0$ & $F_{1,102}=5.8, P<0.05$ & $<0.05$ \\
\hline Pacifier & $7.5 \pm 1.1$ & $6.1 \pm 0.6$ & $F_{1,101}=6.8, P<0.01$ & $<0.05$ \\
\hline
\end{tabular}

${ }^{a} P$-values are for the 5-min Apgar complication scale. Additional significant effects of confounders are not presented in this table
Specific delivery complications. The $0-1$ optimality variables were analyzed separately with ANOVA's, $t$ tests or Mann-Whitneys. Although several ANOVA's additionally displayed significant effects of confounding variables (i.e., "minutes since last feeding," "age in days," etc.), only the significances due to the optimality variables will be discussed below. The following results were found:

Physical examination. The duration of the first stage of labor did not display any relations with the infants' behavioral variables of the physical examination. The duration of the second stage was positively related to being soothed longer by a pacifier/ finger, estimated marginal mean \pm SE: $9.0 \pm 1.2 \%$ of the examination time for pushing longer than $1.5 \mathrm{~h}$ vs. $5.7 \pm 0.6 \%$ for pushing shorter than $1.5 \mathrm{~h}$ (ANOVA: $F_{1,101}=7.7, P=0.001$, effect "duration of the second stage": $P=0.01$ ).

The fetal heart function was related to the latency to begin to cry during the physical examination ( $T$ test; $t=2.0, P=0.05$, for the residuals of cry latency against minutes awake), with the infants with nonreassuring patterns showing shorter latencies to cry (estimated marginal mean \pm SE of cry latency: $373.1 \pm 99.6 \mathrm{~s})$ than the infants with normal patterns $(583.5 \pm 46.8 \mathrm{~s})$.

The significant relations between the behavioral reactions to the physical examination and the 5-min Apgar score are presented in Table 3. As can be seen from the table, infants with 5-min Apgars of less than 10 displayed more irritability and less soothability during the physical examination.

Daily behavior. The only variable that was significantly related to the infants' 6-week behavior was fetal heart function. Infants with non-reassuring fetal heart rate patterns showed longer crying bouts than infants with normal patterns (estimated marginal mean \pm SE: $17.4 \pm 1.9 \mathrm{~min} \quad$ vs. $12.2 \pm 0.9 \mathrm{~min} ; \quad$ ANOVA: $F_{1,68}=3.4, P=0.01$, effect "heart rate": $P<0.05$ ). However, the mean daily duration of fussing was lower in infants with non-reassuring patterns than in those with normal patterns (mean \pm SE: $72.8 \pm 9.2$ min vs.
Table 4 Descriptive values of the untransformed cortisol variables (stress and recovery) according to delivery mode

\begin{tabular}{|c|c|c|c|c|c|c|}
\hline \multirow[b]{3}{*}{ Cortisol variables } & \multicolumn{6}{|c|}{ Delivery mode } \\
\hline & \multicolumn{2}{|c|}{$\begin{array}{l}\text { Vaginal } \\
(n=68)\end{array}$} & \multicolumn{2}{|c|}{$\begin{array}{l}\text { Assisted } \\
(n=34)\end{array}$} & \multicolumn{2}{|c|}{$\begin{array}{l}\text { Emergency } \\
\text { caesarean } \\
(n=12)\end{array}$} \\
\hline & Mean & SD & Mean & SD & Mean & SD \\
\hline Physical exam stress & 7.6 & 9.4 & 5.9 & 12.4 & 6.9 & 12.4 \\
\hline Physical exam recovery & 4.1 & 7.6 & 4.1 & 11.3 & 3.3 & 7.7 \\
\hline Inoculation stress & 6.8 & 10.9 & 4.6 & 9.2 & 4.2 & 10.5 \\
\hline
\end{tabular}

$90.3 \pm 4.7 \mathrm{~min}$; ANOVA: $F_{1,99}=3.9, P=0.05$, effect "heart rate": $P=0.05$ ), and there was a trend in the same direction for mean daily frequency of fussing $\left(4.9 \pm 0.8\right.$ vs. $5.9 \pm 0.4$; ANOVA: $F_{1,99}=2.8, P=0.10$, effect "heart rate": $P=0.10$ ).

Inoculation. The 5-min Apgar score was the only variable related to the infants' behavioral reaction to the inoculation. Infants with a 5-min Apgar score of less than 10 were found to cry more than those with a score of 10 (estimated marginal mean \pm SE: $4.5 \pm 0.2$ vs. $3.9 \pm 0.1$; ANOVA: $F_{1,77}=5.1, P<0.01$, effect "5 min Apgar": $P<0.05)$, and to be more unsoothable post-inoculation (respective mean ranks MannWhitney $U$ test: 54.7 and 41.2, $Z=-2.3, P<0.05)$.

\section{Childbirth complications and cortisol reactivity}

Delivery mode. The ANOVA models for the cortisol reactivity to the physical examination were significant, but only due to confounders (i.e., "minutes since last feeding," "age in days") and with no significant effects of delivery mode on stress and recovery cortisol. Table 4 presents the means and standard deviations of the untransformed cortisol variables (stress and recovery) according to mode of delivery.

Specific delivery complications. The $0-1$ optimality variables were analyzed separately with ANOVA's. Again, although several ANOVA's were significant due to confounders, no significant relations were found between the optimality variables and the cor- 
Table 5 Descriptive values of the untransformed cortisol variables (stress and recovery) according to specific delivery complications

\begin{tabular}{|c|c|c|c|c|c|c|c|c|c|c|c|c|c|c|c|c|}
\hline \multirow[b]{4}{*}{ Cristol Variables } & \multicolumn{16}{|c|}{ Specific delivery complications } \\
\hline & \multicolumn{4}{|c|}{$\begin{array}{l}\text { Duration first stage } \\
\text { (dilatation) }\end{array}$} & \multicolumn{4}{|c|}{$\begin{array}{l}\text { Duration second stage } \\
\text { (pushing) }\end{array}$} & \multicolumn{4}{|c|}{$\begin{array}{l}\text { Fetal heart } \\
\text { function }\end{array}$} & \multicolumn{4}{|c|}{$\begin{array}{l}\text { Apgar score, } \\
5 \mathrm{~min}\end{array}$} \\
\hline & \multicolumn{2}{|c|}{$\begin{array}{l}\text { Optimal } \\
(n=86)\end{array}$} & \multicolumn{2}{|c|}{$\begin{array}{l}\text { Less optimal } \\
(n=28)\end{array}$} & \multicolumn{2}{|c|}{$\begin{array}{l}\text { Optimal } \\
(n=89)\end{array}$} & \multicolumn{2}{|c|}{$\begin{array}{l}\text { Less optimal } \\
(n=25)\end{array}$} & \multicolumn{2}{|c|}{$\begin{array}{l}\text { Optimal } \\
(n=96)\end{array}$} & \multicolumn{2}{|c|}{$\begin{array}{l}\text { Less optimal } \\
(n=20)\end{array}$} & \multicolumn{2}{|c|}{$\begin{array}{l}\text { Optimal } \\
(n=89)\end{array}$} & \multicolumn{2}{|c|}{$\begin{array}{l}\text { Less optimal } \\
(n=27)\end{array}$} \\
\hline & Mean & SD & Mean & SD & Mean & SD & Mean & SD & Mean & SD & Mean & SD & Mean & SD & Mean & SD \\
\hline Physical exam stress & 6.9 & 10.6 & 7.4 & 10.6 & 6.7 & 9.9 & 8.4 & 12.7 & 6.3 & 9.7 & 10.7 & 13.8 & 7.8 & 11.5 & 4.7 & 6.2 \\
\hline Physical exam recovery & 4.2 & 9.1 & 3.5 & 7.7 & 3.4 & 8.3 & 6.2 & 10.2 & 3.6 & 8.0 & 6.0 & 11.9 & 4.2 & 9.4 & 3.5 & 6.8 \\
\hline Inoculation stress & 5.2 & 10.8 & 7.7 & 7.9 & 5.5 & 10.8 & 6.4 & 8.5 & 5.6 & 10.0 & 6.5 & 11.7 & 5.4 & 9.9 & 6.6 & 11.2 \\
\hline
\end{tabular}

tisol reactions to the stressors. Table 5 shows the means and standard deviations of the untransformed cortisol variables (stress and recovery) according to the optimality of the delivery complications.

\section{Discussion}

\section{Childbirth and infant behavior}

In the present longitudinal study, the presence of childbirth complications was in general related to more crying, fussing and unsoothability, especially in situations that were stressful to the infant. In other words, healthy infants born after slightly less optimal, albeit uncomplicated deliveries appeared to be in general more arousable or reactive in the expression of negative emotions, and to have more problems regulating their negative affect. Although the size of the associations was modest, the effects extended over different types of situations, and until the age of 2 months. The results are in line with our hypothesis and support earlier findings $[11,12]$. However, it is not possible from this study to conclude that there is a direct causal link between stressful childbirth events and infant irritability in behavior. One cannot rule out alternative scenarios such as for example, that childbirth complications cause changes in maternal behavior that in turn influence infant behavior. Also, as we had expected, specific delivery complications were more strongly related to subsequent infant behavior than mode of delivery. This was especially the case with the Apgar score and fetal heart rate, and shows that these specific complications are more adequate indicators of the fetal birth stress than delivery mode and duration of delivery stages, probably because they are more closely related to fetal and neonatal status.

The only unexpected finding was that while nonreassuring fetal heart function was related to longer 6week crying bouts, it was also related to shorter daily fussing duration, and showed a tendency for lower fussing frequency. A possible explanation for this could be that infants who engage in longer crying bouts, spend less time fussing and fuss less frequently. In other words, infants with more problems regulating their negative emotionality (i.e., longer crying bouts) would not necessarily be those that show more emotional reactivity (i.e., more frequent fussing). Indeed, post-hoc analyses indicated that the infants' mean length of the crying bouts was negatively correlated to their frequency of fussing $(r=-0.21, P=0.03)$, and positively correlated to their mean length of the fussing bouts $(r=0.55, P<0.001)$, hinting at a trade-off between bout length and frequency that could explain our fetal heart function results.

\section{Childbirth and infant cortisol reactivity}

The two stressors used in this study produced significant cortisol reactions, which is normal for these infant ages and stressors. Nonetheless, no relations were found between delivery mode and specific complications, and the infants' cortisol reactions to the stressors. This supports the findings of Keenan et al. [11] and Miller et al. [16], but is in contrast to those of Taylor et al. [24], for vaginal and assisted deliveries, and of Ramsay and Lewis [19]. Since we did not have enough elective caesarean sections, we were unable to compare our findings to those of Taylor et al. in this respect.

Our negative results for the cortisol data could be due to the important amount of variability in the cortisol reactivity data. Infants differed greatly in the magnitude of their cortisol reaction to the stressor, were not individually stable in their reactions to both stressors, and did not show positive correlations between their cortisol and behavioral reactions to the stressors. This is in line with earlier findings of large intra-individual variability in infant basal cortisol [3, 5]. The fact that the stressors produced significant cortisol reactions, and that we controlled for a variety of possible confounders, points at the instability and 
variability in cortisol levels being normal, and not due to validity problems. In turn, this variability in cortisol could make it more difficult to find clear relations between childbirth complications and subsequent cortisol physiology, and could explain the differences in results between the studies.

Another possible explanation for the lack of a link between mode of delivery and the cortisol reactions to the inoculation is that parity is a confounding factor. In our study, all infants were firstborn. In the study by Taylor et al. [24] for example, the parity is not reported, opening the possibility that infants of different parities participated. If the firstborns were more often delivered by assisted deliveries than for example second or third children, then this could be a confounding factor that makes the results of both studies incomparable. Indeed, there is evidence that primiparous women have both twice as high levels of maternal cortisol during childbirth, as more often emergency caesarean deliveries, than multiparous women [7]. Further giving weight to this hypothesis, as stated earlier, Miller and colleagues [16] did not replicate their earlier findings on delivery mode and cortisol reactivity [24] in their larger study, and this study was performed solely on primimarae. Therefore, the effects of the mode of delivery on the fetus could be different depending on the parity of the mother, and this factor should be controlled for in future studies.

Finally, it is possible that only childbirth complications, which are associated with more severe fetal stress have programming effects on the infant's subsequent stress physiology. In the present study we looked at the effects of less optimal delivery factors within the normal variations of uncomplicated, term deliveries.

\section{Additional points and conclusions}

An interesting secondary finding of the study was that the infants' cortisol and behavioral variables were mostly significantly correlated to one or several situational confounders (i.e., hunger, tiredness, sleep, age, time of day) and birth outcome confounders (i.e., duration of gestation, weight, length, head circumference). This underlines the importance of taking these confounding factors into account when studying infant behavior and cortisol.

A limitation of the current study is that due to multiple statistical analyses, the possibility of chance findings is increased. Future replication studies are therefore necessary. However, the significant results were many and mainly in the same direction, even after correcting for confounders. This makes us confident that they most probably reflect actual relationships between childbirth and later development.

More research is necessary to determine how long these apparent influences of childbirth on infant behavior last, and whether they become stronger with more serious complications. Also, it would be interesting to determine whether these effects have a greater or lesser impact on development according to the type of social environment the infant is born into.

Our findings allow us to conclude that in relatively uncomplicated deliveries with normal outcome, slightly less optimal childbirth characteristics appear to be linked to more crying/fussing in the infant and more difficulties regulating this behavior, but not to infant cortisol reactivity to stressors. The effects apparently extend over different types of situations, and at least until the age of 2 months. Childbirth complications should therefore be taken into account in studies on early infant behavior.

\begin{abstract}
- Acknowledgements The authors wish to thank the families that participated in the study, and J. Jansen for his helpful comments on an earlier version of this manuscript. This research was supported by the Netherlands Organization for Scientific Research (NWO), Grant number 575-25-009, and was carried out at the Department of Child and Adolescent Psychiatry of the University Medical Center of Utrecht, The Netherlands, where the authors were formerly affiliated.
\end{abstract}

\section{References}

1. Barr RG, Kramer MS, Boisjoly C, McVey-White L, Pless IB (1988) Parental diary of infant cry and fuss behaviour. Arch Dis Childhood 63:380387

2. de Weerth C, van Geert P, Hoijtink H (1999) Intra-individual variability in infant behavior. Dev Psychol 35:11021112
3. de Weerth C, van Geert P (2002) A longitudinal study of basal cortisol in infants: Intra-individual variability, circadian rhythm and developmental trends. Infant Behav Dev 25:375-398

4. de Weerth C, Graat G, Buitelaar JK, Thijssen JH (2003) Measurement of cortisol in small quantities of saliva. Clin Chem 49:658-660
5. de Weerth C, Zijl RH, Buitelaar JK (2003) Development of cortisol circadian rhythm in infancy. Early Hum Dev 73:39-52

6. Gitau R, Menson E, Pickles V, Fisk NM, Glover V, MacLachlan N (2001) Umbilical cortisol levels as an indicator of the fetal stress response to assisted vaginal delivery. Eur J Obstet Gynecol Reprod Biol 98:14-17 
7. Grajeda R, Perez-Escamilla R (2002) Stress during labor and delivery is associated with delayed onset of lactation among urban Guatemalan women. J Nutr 132:3055-3060

8. Gunnar MR, Nelson CA (1994) Eventrelated potentials in year-old infants: relations with emotionality and cortisol. Child Dev 65:80-94

9. Gunnar MR, Brodersen L, Krueger K, Rigatuso J (1996) Dampening of adrenocortical responses during infancy: normative changes and individual differences. Child Dev 67:877-889

10. Hadders-Algra M, Huisjes HJ, Touwen BC (1988) Perinatal correlates of major and minor neurological dysfunction at school age: a multivariate analysis. Dev Med Child Neurol 30:472-481

11. Keenan K, Grace D, Gunthorpe D (2003) Examining stress reactivity in neonates: relations between cortisol and behavior. Child Dev 74:1930-1942

12. Keller H, Lohaus A, Volker S, Cappenberg M, Chasiotis A (1998) Relationships between infant crying, birth complications, and maternal variables. Child Care Health Dev 24:377-394

13. Lewis M, Ramsay DS (1995) Developmental change in infants' responses to stress. Child Dev 66:657-670

14. Lewis M, Ramsay D (2005) Infant emotional and cortisol responses to goal blockage. Child Dev 76:518-530
15. Linden W, Earle TL, Gerin W, Christenfeld N (1997) Physiological stress reactivity and recovery: conceptual siblings separated at birth? J Psychosom Res 42:117-135

16. Miller NM, Fisk NM, Modi N, Glover V (2005) Stress responses at birth: determinants of cord arterial cortisol and links with cortisol response in infancy. BJOG 112:921-926

17. Pohjavuori M, Rovamo L, Laatikainen T (1985) Plasma immunoreactive betaendorphin and cortisol in the newborn infant after elective caesarean section and after spontaneous labour. Eur J Obstet Gynecol Reproduct Biol 19:6774

18. Prechtl HFR (1977) The neurological examination of the full-term newborn infant. Clinics in developmental medicine, nr. 63. Lavenham Press, Suffolk

19. Ramsay DS, Lewis M (1995) The effects of birth condition on infants' cortisol response to stress. Pediatrics 95:546549

20. Roy MP (2004) Patterns of cortisol reactivity to laboratory stress. Hormones Behav 46:618-627

21. Soorani-Lunsing RJ, Hadders-Algra $M$, Huisjes HJ, Touwen BC (1993) Minor neurological dysfunction after the onset of puberty: association with perinatal events. Early Hum Dev 33:71-80
22. Taddio A, Katz J, Ilersich AL, Koren G (1997) Effect of neonatal circumcision on pain response during subsequent routine vaccination. Lancet 349:599603

23. Taddio A, Shah V, Gilbert-MacLeod C, Katz J (2002) Conditioning and hyperalgesia in newborns exposed to repeated heel lances. JAMA 288:857-861

24. Taylor A, Fisk NM, Glover V (2000). Mode of delivery and subsequent stress response. Lancet 355:120

25. Thijssen JHH, Van den Berg JHM, Adlercreutz H, Gijzen AHJ, de Jong FH, Moolenaar AJ (1980) The determination of cortisol in human plasma: evaluation and comparison of seven assays. Clin Chim Acta 100:39-46

26. van den Berg A, van Elburg RM, van Geijn HP, Fetter WP (2001) Neonatal respiratory morbidity following elective caesarean section in term infants. A 5-year retrospective study and a review of the literature. Eur J Obstet Gynecol Reproduct Biol 98:9-13

27. Ziadeh SM, Sunna E (2000) Obstetric and perinatal outcome of pregnancies with term labour and meconiumstained amniotic fluid. Arch Gynecol Obstet 264:84-87 